\title{
A rapid and fully automatic method for the accurate determination of a wide carbon-chain range of per- and polyfluoroalkyl substances (C4-C18) in human serum
}

\author{
Ke Gao, Yan Gao, Yili Li, Jianjie Fu*, Aiqian Zhang* \\ State Key Laboratory of Environmental Chemistry and Ecotoxicology, Research Center for Eco-Environmental Sciences, Chinese Academy of Sciences, Beijing \\ 100085, China
}

\section{A R T I C L E I N F O}

\section{Article history:}

Received 5 July 2016

Received in revised form

18 September 2016

Accepted 22 September 2016

Available online 23 September 2016

\section{Keywords:}

Fully automatic

On-line turboflow SPE

PFASs

Short- and long-chain PFASs

Human serum

\begin{abstract}
A B S T R A C T
A rapid and fully automatic method for determining 21 per- and polyfluoroalkyl substances (with carbon chains ranging from $\mathrm{C} 4$ to $\mathrm{C} 18$, including 13 PFCAs, 5 PFSAs, 2 Cl-PFESAs, and PFOSA) in human serum samples was developed. The HPLC parameters, Turboflow column, mobile phase, sample injection volume, loading flow rate, and sample cleanup and elution time were optimized. $25 \mu \mathrm{L}$ serum sample was directly injected into the developed on-line Turboflow SPE HPLC-MS/MS system for analysis after dilution. Matrix effects were corrected due to the matrix removal efficiency of the Turboflow column and sufficient types of internal isotope standards that were used. The established method showed a good linearity $\left(r^{2}>0.99\right)$, rapid processing time ( 20 min per sample), satisfactory recoveries (matrix spiked recoveries range from $84.6 \%$ to $114 \%$ ) and precision (intra-day and inter-day RSDs ranged from $1.5 \%$ to $9.2 \%$ and from $1.1 \%$ to $7.0 \%$, respectively). The limits of detection (LODs) of the 21 analyzed PFASs were between 0.008 and $0.19 \mathrm{ng} \mathrm{mL}^{-1}$. The LODs of short- and long-chain PFASs, such as PFBA, PFPeA, PFHxDA, and PFODA, were 0.008, 0.022, 0.15 and $0.19 \mathrm{ng} \mathrm{mL}^{-1}$, respectively; the spiked recoveries of these PFASs were $101,105,87.1$, and $85.8 \%$, respectively. Both the LODs and recoveries were better than previous studies. Further, serum PFASs concentrations detected by the presented on-line SPE method were consistent with the traditional off-line SPE method ( $r$ : 0.98-0.99), which verified the accuracy and applicability of the present method. The method shows good practical prospects in the analysis of trace per- and polyfluoroalkyl substances in human serum.
\end{abstract}

(c) 2016 Elsevier B.V. All rights reserved.

\section{Introduction}

Per- and polyfluoroalkyl substances (PFASs) are compounds with unique physicochemical properties. These compounds have been produced and used for many different industrial and commercial applications, such as textiles, paper packaging, carpets, pesticides, surfactants, polymer additives and fire-fighting foams, since the 1950s [1-3]. PFASs have been detected in human serum [4-6], milk [7], and urine [8]. Their potential risks, such as reproductive and developmental toxicity to humans, have caused extensive public concern $[9,10]$. Although most leading global fluorochemical manufacturing companies have agreed to phase out perfluorooctanoate (PFOA), perfluorooctane sulfonate (PFOS) and related chemicals and the concentrations of PFOS in humans are apparently

\footnotetext{
* Corresponding authors.

E-mail addresses: jjfu@rcees.ac.cn (J. Fu), aqzhang@rcees.ac.cn (A. Zhang).
}

decreasing over time [6,11], numerous nonregulated alternative PFASs are being produced in increasing quantities [12,13].

For example, short-chain PFASs, such as perfluorobutanoic acid (PFBA) (C4), perfluorobutanesulfonate (PFBS) (C4), have been used as alternatives for PFOA and PFOS by several manufacturers [12]. Pérez et al. found a high concentration of short-chain PFASs in human lungs, which indicated that more attention should be paid to these compounds [17]. Additionally, polyfluoroalkyl substances, such as $6: 2$ chlorinated polyfluorinated ether sulfonate $(6: 2 \mathrm{Cl}$ PFESA, with the trade name F-53B), have been applied as a mist suppressant in hard chrome plating for more than 30 years in China $[14,15]$. F-53B may have bioaccumulation potential and toxic effects because of its structural similarities with PFOS [2,14,16]; however, there was only one study regarding the concentrations of F-53B in human serum to date [16] and more data were needed.

Several studies found the increasing temporal trends of longchain PFASs in Chinese sturgeon and human blood $[18,19]$. Moreover, the greater bioaccumulative potential and toxicities of 
long-chain PFASs were also reported [20,21]. The carbon chain length of long-chain PFASs in these studies were generally less than 12, more data for PFASs with carbon chain length longer than 12 (such as perfluorotetradecanoic acid (PFTeA) (C14), perfluorohexadecanoic acid (PFHxDA) (C16) and perfluorooctadecanoic acid (PFODA) (C18)) was needed to further clarify their toxic potencies [22].

High performance liquid chromatography coupled with tandem mass spectrometry (HPLC-MS/MS) is the most common method for analyzing these compounds [4,5,16,23-26], which has good sensitivity and low detection limits for PFASs. Liquid-liquid extraction (LLE) was performed with an ion-pairing agent, such as tetra- $n$ butylammonium hydrogen sulfate (TBA), and then extracted using methyl tert-butyl ether (MTBE), followed by solid phase extraction (SPE); the extracted liquid is generally used as a pre-treatment to extract and pre-concentrate PFASs from serum samples [4-6,16,23] and was regard as an off-line SPE method for the determination of PFASs in human serum. However, the off-line SPE method was time-consuming and solvent-intensive and may introduce errors during the manual pre-treatment process. On-line SPE methods could overcome these shortcomings mentioned above, till now, several on-line SPE coupled with LC-MS methods have been applied to the analysis of PFAS in serum. At present, the reported online SPE methods require the precipitation of the protein in serum samples before injecting the sample into the analytical instruments $[24,25,27]$, and no fully automatic on-line method for the analysis of PFASs in serum was available. Moreover, previous studies usually focused on PFASs of a moderate carbon chain length (C6-C10). The polarity and hydrophilicity of the PFASs varied with the carbon chain length and the different functional groups; therefore, simultaneously detecting a wide range of PFASs (C4-C18) was a challenge, especially for shorter (PFBA, perfluoropentanoic acid (PFPeA), and PFBS) and longer chain PFASs (PFHxDA and PFODA). PFBA, PFPeA and PFBS usually showed poor chromatographic peak intensities or unsatisfactory recoveries during the analytical process; PFHxDA and PFODA usually had low spiked recoveries (less than $50 \%$ ), which made them difficult to determine $[1,23,28]$.

Investigations of PFAS contamination involving humans usually involve a large number of samples, and therefore, a rapid, accurate and fully automatic method, which has the capacity for direct injection, is urgently needed. Ideally, this method could simultaneously detect the wide range of PFASs mentioned above. Turboflow column is a novel type of column for on-line extraction and pre-concentration. The turbulent fluid in the column can provide an efficient separation of large matrix components from smaller molecules and has been applied to the analysis of PFASs in urine and water samples $[26,29]$. However, it has not yet been used for the analysis of PFASs in human serum. The matrix of human serum is far more complex than that of water and urine. Serum samples contain compounds with a wide range of molecular weights, such as proteins, peptides, fats, carbohydrates, hormones and inorganics [30]. If the complex matrix in serum can be removed by optimizing the on-line Turboflow SPE parameters, the Turboflow SPE technology could be used to establish a rapid and fully automatic on-line method.

The aim of the present study is to develop a rapid, accurate, and fully automatic method without any sample pre-treatment for analyzing a wide range of PFASs in human serum. The Turboflow SPE column, sample volume, loading flow rate, sample cleanup and elution time were carefully optimized in this study. In addition to optimizing these on-line Turboflow SPE HPLC parameters, we employed as many internal isotope standards as possible to correct the matrix effects; a total of 13 types of isotope-labeled PFASs were used in the present study, including a new commercial internal standard, $\left[{ }^{13} \mathrm{C}_{2}\right]$-perfluorobutane sulfonate (MPFBS). Finally, the established method was then applied to human serum samples $(n=35)$ and verified using the traditional off-line SPE method.

\section{Experimental section}

\subsection{Chemicals and materials}

PFAC-MXB (98\% purity in methanol) containing PFBA, PFPeA, perfluorohexanoic acid (PFHxA), perfluoroheptanoic acid (PFHpA), PFOA, perfluorononanoic acid (PFNA), perfluorodecanoic acid (PFDA), perfluoroundecanoic acid (PFUdA), perfluorododecanoic acid (PFDoA), perfluorotridecanoic acid (PFTrA), PFTeA, PFHxDA, PFODA, PFBS, perfluorohexanesulfonate (PFHxS), PFOS, and perfluorodecanesulfonate (PFDS); perfluoroheptanesulfonate (PFHpS); perfluorooctane sulphonic acid (PFOSA); MPFAC-MXA (>98\%) containing $\left[{ }^{13} \mathrm{C}_{4}\right]$-perfluorobutanoic acid (MPFBA $\left.\left({ }^{13} \mathrm{C}_{4}\right)\right), \quad\left[{ }^{18} \mathrm{O}_{2}\right]$-perfluorohexanesulfonate (MPFHxS $\left.\left({ }^{18} \mathrm{O}_{2}\right)\right), \quad\left[{ }^{13} \mathrm{C}_{2}\right]$-perfluorohexanoic acid (MPFHxA $\left.\left({ }^{13} \mathrm{C}_{2}\right)\right)$, $\left[{ }^{13} \mathrm{C}_{4}\right]$-perfluorooctanesulfonate (MPFOS $\left({ }^{13} \mathrm{C}_{4}\right)$ ), $\left[{ }^{13} \mathrm{C}_{4}\right]-$ perfluorooctanoic acid (MPFOA $\left.\left({ }^{13} \mathrm{C}_{4}\right)\right),\left[{ }^{13} \mathrm{C}_{5}\right]$-perfluorononanoic acid (MPFNA $\left.\left({ }^{13} \mathrm{C}_{5}\right)\right)$, $\left[{ }^{13} \mathrm{C}_{2}\right]$-perfluorododecanoic acid (MPFDoA $\left.\left({ }^{13} C_{2}\right)\right),\left[{ }^{13} C_{2}\right]$-perfluorodecanoic acid (MPFDA $\left.\left({ }^{13} C_{2}\right)\right)$, and $\left[{ }^{13} \mathrm{C}_{2}\right]$-perfluoroundecanoic acid (MPFUdA $\left({ }^{13} \mathrm{C}_{2}\right)$ ); $\left[{ }^{13} \mathrm{C}_{4}\right]-$ perfluoropentanoic acid (MPFPeA); $\left[{ }^{13} \mathrm{C}_{2}\right]$-perfluoroheptanoic acid (MPFHpA); MPFBS; and $\left[{ }^{13} \mathrm{C}_{8}\right]$-perfluorooctanesulfonate (MPFOS $\left({ }^{13} C_{8}\right)$ ) were supplied by Wellington Laboratories, Canada. 6:2 Cl-PFESA and 8:2 Cl-PFESA were purified from the commercial F-53 B product purchased from Shanghai Synica Co., Ltd. LC-MSgrade solvents, acetonitrile, methanol, ammonium hydroxide (28\%) were purchased from Fisher Scientific (Ottawa, ON, Canada). Ammonium acetate $\left(\mathrm{NH}_{4} \mathrm{OAc},>97 \%\right)$, acetic acid $(>99.8 \%$, HPLC grade), and formic acid ( $>98 \%$, HPLC grade) were obtained from Alfa Aesar (Ward Hill, MA, USA). Water $(>18.2 \mathrm{MV} \mathrm{cm})$ used in the present study was purified on a Milli-Q Advantage A10 system (Millipore, USA).

\subsection{Instrumentation}

The UltiMate ${ }^{\mathrm{TM}} 3000$ system (Thermo Scientific, USA) consisted of a dual- gradient rapid separation pump DGLC-3600RS, a TCC3200 column oven with a two-position, six-port (2P-6P) valve, and a WPS-3000 TLS auto-sampler. The entire analysis process is controlled by a Chromeleon 6.70 chromatography workstation. A TSQ Quantiva triple quadrupole mass spectrometer (Thermo Scientific, USA) was used to measure the target compounds. The data was recorded using the Xcalibur 3.0 software. The schematic of the on-line Turboflow-SPE-HPLC-MS/MS system is shown in Fig. S1 in Supplementary materials.

\subsection{On-line SPE procedure, HPLC analysis and MS detection}

The on-line SPE procedure included sample pre-concentration, cleanup, elution, SPE column regeneration, and HPLC separation steps. The mobile phase consisted of $0.1 \%$ formic acid in water $(A)$, acetonitrile $(B)$, distilled water $(C)$, delivered by left pump; $30 \mathrm{mmol} \mathrm{L}^{-1}$ ammonium formate buffer solution in water $(\mathrm{A})(\mathrm{pH}$ adjusted to 4 ) and acetonitrile (B), distilled water (C), delivered by right pump. Initially, the valve of on-line SPE liquid chromatography was switched to the loading position, shown in Fig. S1(a) in Supplementary materials. Due to the high viscosity, serum was diluted with distilled water firstly, then each $25 \mu \mathrm{L}$ serum dilution was drawn by a syringe from a $250 \mu \mathrm{L}$ liner pipe and injected into the large-volume loop, which was carried over by the mobile phase from left pump (loading pump) and then delivered to the on-line SPE column (Turboflow Cyclone-P, $1.0 \times 50 \mathrm{~mm}$, Thermo Scientific) at a high mobile-phase flow rate $\left(2 \mathrm{~mL} \mathrm{~min}^{-1}, 100 \% \mathrm{~A}\right)$. 
Initial mobile phase of loading pump $\left(2 \mathrm{~mL} \mathrm{~min}^{-1}, 100 \% \mathrm{~A}\right)$ was kept for 1.0 min to clean up the matrix components that concentrated on

Recoveries \%

PFASs concentrations detected in spiked serum sample - PFASs concentrations detected in unspiked serum sample Theoretical PFASs concentration in spiked serum sample

$\times 100 \%$

the SPE column. Noteworthy that to on-line Turboflow SPE column was needed to be regenerated after the elution step, thus loading

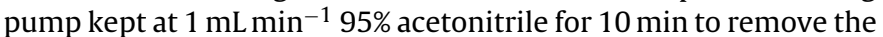
residual contamination and ensure method reproducibility. And then the mobile phase of loading pump was returned to its original composition for the next sample.

After sample cleanup, the valve was switched to the injecting position (Fig. S1(b) in Supplementary materials) to elute the analytes from the SPE column to the analytical column and maintained for $5.0 \mathrm{~min}$. The trapped analytes were desorbed from the Turboflow column and eluted on to the analytical column for further separation and detection. Then the valve returned to the loading position (Fig. S1(a) in Supplementary materials), while the eluted analytes were further separated on the analytical column (Acclaim $120 \mathrm{C} 18,4.6 \times 150 \mathrm{~mm}, 5.0 \mu \mathrm{m}$, Thermo Scientific) at a flow rate of $0.8 \mathrm{~mL} \mathrm{~min}^{-1}$. The analytical column temperature was set at $40^{\circ} \mathrm{C}$. The on-line SPE procedure and HPLC gradient elution conditions for the left and right pumps and the timing of the valve switching are listed in Table 1.

The mass spectrometer was operated in the negative ion ESI-MS/MS using the Multiple Reaction Monitoring (MRM) mode. The MS related parameters were set as follows: sheath gas, 40 units; aux gas, 12 units; source voltage, $2500 \mathrm{~V}$; vaporizer temperature, $350^{\circ} \mathrm{C}$; capillary temperature, $400^{\circ} \mathrm{C}$; and scan time, $0.01 \mathrm{~s}$. Other parameters and analyzed compounds are listed in Table 2 in Supplementary materials.

\subsection{Method validation, quality control and quality assurance}

The data acquisition and analysis for all of the samples, blanks, standards, and QC materials were performed using the analysis software of the Xcalibur 3.0, Foundation 3.0 (Thermo Scientific, USA). The developed method was validated using standard solutions, internal isotope standards and real serum samples to evaluate the linearity, selectivity, accuracy, precision, recovery and matrix effects, limits of detection (LODs) and limits of quantification (LOQs).

\subsection{Serum samples collection and preparation}

Serum samples $(n=35)$ were collected from workers of a fluorochemical manufacturing facility $(n=20)$ and ordinary people $(n=15)$ in Wuhan, Hubei Province in 2013. After sampling, serum was separated from red blood cells and other components by centrifugation at $3000 \mathrm{rpm}$ for $10 \mathrm{~min}$. Then, the serum samples were transferred as soon as possible to the laboratory in polypropylene containers and stored at $-20^{\circ} \mathrm{C}$ until analysis. Because the human serum was too thick to draw accurately and might clog the syringe, the serum samples were diluted with distilled water before injection. Briefly, $25 \mu \mathrm{L}$ serum sample was diluted by $175 \mu \mathrm{L}$ mixture of distilled water and isotope internal standards solution, and then $25 \mu \mathrm{L}$ serum dilution was directly injected to LC-MS/MS by the auto-sampler. To verify the results of the present study, the traditional off-line SPE method was also used to analyze the serum samples. Detailed information about the off-line SPE method was included in Supplementary materials. In the present study, spiked recoveries of detected PFASs were calculated by Eq. (1), and the
PFASs concentrations below LOD in unspiked serum were set as LOD/2 when calculated spiked recoveries.
Two serum samples were selected for the calculation of LODs in the present study: one sample did not contain target analytes except for PFBS $\left(0.18 \mathrm{ng} \mathrm{mL}^{-1}\right)$, and another serum sample did not contain PFBS. The LODs of PFASs were obtained by spiking a serial of low concentrations standard solutions (ranging from $0.005 \mathrm{ng} \mathrm{mL}^{-1}$ to $0.5 \mathrm{ng} \mathrm{mL} \mathrm{m}^{-1}$ ) into the serum samples and expressed as a concentration at signal-to-noise ratio equal 3:1 in the present study.

\section{Results and discussion}

\subsection{Optimization of the on-line Turboflow SPE HPLC procedure}

To obtain the optimized chromatographic efficiency, the trapped analytes should be eluted and refocused onto the analytical column when the extraction column was switched to the analytical flow path. Several related conditions of on-line Turboflow SPE HPLC procedure, such as the HPLC parameters, selection of the on-line SPE column (Turboflow Cyclone-P column), sample volume $(25 \mu \mathrm{L})$, sample loading flow rate $\left(2 \mathrm{~mL} \mathrm{~min}^{-1}\right)$, and sample cleanup ( $1 \mathrm{~min}$ ) and elution time ( $5 \mathrm{~min}$ ), were carefully optimized in the present study.

\subsubsection{HPLC parameters}

The HPLC parameters were optimized to ensure the identification and quantification of all selected analytes. The Acclaim $120 \mathrm{C} 18$ column $(4.6 \times 150 \mathrm{~mm}, 5.0 \mu \mathrm{m}$, Thermo Scientific) was selected as the analytical column which has been applied to the separation and detection of PFASs in our previous studies [4,5]. A guard column $(4.6 \times 50 \mathrm{~mm}, 5.0 \mu \mathrm{m}$, Thermo Scientific) was placed between the analytical pump and injection valve to decrease the interfering background peaks of the PFASs. The mobile phase was then optimized using the above selected columns. Better peak shapes for the analytes, especially PFBA and PFPeA, were obtained using the acetonitrile rather than methanol (Fig. 1(a)). Ammonium formate buffer ( $\mathrm{pH}$ adjusted to 4 ) were optimized and applied to detect PFASs in previous studies $[4,5,24,34]$. Five different concentrations $\left(5,10,20,30,40 \mathrm{mmol} \mathrm{L}^{-1}\right)$ of ammonium formate buffer were tested on the separation, peak shape, and sensitivity for PFASs determination ( $\mathrm{pH}$ maintained at 4 ) in the present study. Increasing the concentration of ammonium formate from 5 to $30 \mathrm{mmol} \mathrm{L}^{-1}$ resulted in sharper, more symmetrical peaks, and higher resolution and sensitivity for the PFASs. However, when the concentration of ammonium formate was increased to $40 \mathrm{mmol} \mathrm{L}^{-1}$, the detection sensitivities for most of the PFASs did not improve and some even worse, it might due to high mobile phase buffer concentration exhibited effects on ionization efficiency (or sensitivity), and thus resulted in a severe suppression of the analyte signal intensity. Therefore, $30 \mathrm{mmol} \mathrm{L}^{-1}$ ammonium formate was selected for the further study. Different percentages of acetonitrile in initial mobile phase were also optimized. More attention was paid to short-chain PFASs (PFBA, PFPeA) in the present study, they were higher water solubility and weaker sorption potentials than longchain PFASs [31]. Peak shapes and intensities of short-chain PFASs were improved when the percentage of acetonitrile was increased from $5 \%$ to $10 \%$ (Fig. 1(b)). The low percentage of acetonitrile (5\%) resulted in long retention time and poor peak shape due to its weak elution ability. However, the peak shape and intensity of PFBA became worse when the percentage of acetonitrile increased from 
Table 1

On-line SPE procedure, HPLC gradient elution and valve switching program.

\begin{tabular}{|c|c|c|c|c|c|c|c|c|c|c|}
\hline \multirow[t]{2}{*}{ Step } & \multirow[t]{2}{*}{ Time (min) } & \multicolumn{4}{|l|}{ left pump } & \multicolumn{4}{|l|}{ Analyzing pump } & \multirow[t]{2}{*}{ Valve position } \\
\hline & & Flow rate $\left(\mathrm{mL} \mathrm{min}{ }^{-1}\right)$ & $\% A$ & $\% \mathrm{~B}$ & $\overline{\% C}$ & Flow rate $\left(\mathrm{mL} \mathrm{min}{ }^{-1}\right)$ & $\% A$ & $\% \mathrm{~B}$ & $\overline{\% C}$ & \\
\hline 1 & 0.0 & 2 & 100 & 0 & & 0.8 & 90 & 10 & & $1-2^{\mathrm{a}}$ \\
\hline 2 & 1.0 & 2 & 100 & 0 & & 0.8 & 90 & 10 & & $6-1^{\mathrm{b}}$ \\
\hline 3 & 6.0 & 1 & 0 & 95 & 5 & 0.8 & 90 & 10 & & $1-2$ \\
\hline 4 & 7.0 & 1 & 0 & 95 & 5 & 0.8 & 55 & 45 & & $1-2$ \\
\hline 5 & 11.0 & 1 & 0 & 95 & 5 & 0.8 & 23 & 77 & & $1-2$ \\
\hline 6 & 14.0 & 1 & 0 & 95 & 5 & 0.8 & 0 & 98 & 2 & $1-2$ \\
\hline 7 & 16.0 & 1 & 0 & 95 & 5 & 0.8 & 0 & 98 & 2 & $1-2$ \\
\hline 8 & 17.0 & 2 & 100 & 0 & & 0.8 & 0 & 98 & 2 & $1-2$ \\
\hline 9 & 17.2 & 2 & 100 & 0 & & 0.8 & 90 & 10 & & $1-2$ \\
\hline 10 & 20.0 & 2 & 100 & 0 & & 0.8 & 90 & 10 & & $1-2$ \\
\hline
\end{tabular}

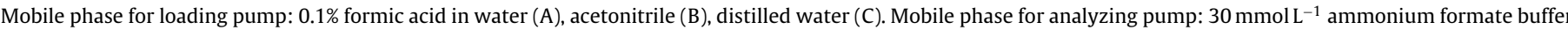
solution in water (A) (pH adjusted to 4), acetonitrile (B), distilled water (C)

a 1-2: loading position.

b 1-6: injecting position.

Table 2

Target analytes measured in the present study with QA/QC information including monitoring parent ion, product ion. Quantifying ions are highlighted in bold.

\begin{tabular}{|c|c|c|c|c|c|}
\hline Compound & Precursor $(m / z)$ & Product $(m / z)$ & Collision Energy (V) & RF Lens (V) & Internal Standard \\
\hline PFBA & 213 & 169 & 8 & 30 & ${ }^{13} \mathrm{C}_{4}$-PFBA \\
\hline PFPeA & 263 & 219 & 10 & 30 & ${ }^{13} \mathrm{C}_{4}$-PFPeA \\
\hline PFHxA & 313 & 269 & 5 & 30 & ${ }^{13} \mathrm{C}_{2}$-PFHxA \\
\hline PFHpA & 363 & 319 & 10 & 47 & ${ }^{13} \mathrm{C}_{2}$-PFHpA \\
\hline PFOA & 413 & 369 & 8 & 39 & ${ }^{13} \mathrm{C}_{4}$-PFOA \\
\hline PFNA & 463 & 419 & 18 & 44 & ${ }^{13} \mathrm{C}_{5}$-PFNA \\
\hline PFDA & 513 & 469 & 17 & 40 & ${ }^{13} C_{2}$-PFDA \\
\hline PFUnA & 563 & 519 & 16 & 52 & ${ }^{13} \mathrm{C}_{2}$-PFUnA \\
\hline PFDoA & 613 & 569 & 10 & 53 & ${ }^{13} \mathrm{C}_{2}$-PFDoA \\
\hline PFTrDA & 663 & 619 & 11 & 61 & ${ }^{13} \mathrm{C}_{2}$-PFDoA \\
\hline PFTeDA & 713 & 669 & 13 & 71 & ${ }^{13} \mathrm{C}_{2}$-PFDoA \\
\hline PFUnDA & 813 & 769 & 13 & 70 & ${ }^{13} \mathrm{C}_{2}$-PFDoA \\
\hline PFODA & 913 & 869 & 13 & 67 & ${ }^{13} C_{2}$-PFDoA \\
\hline PFBS & 299 & $\mathbf{8 0 , 9 9}$ & 33 & 113 & ${ }^{13} \mathrm{C}_{2}$-PFBS \\
\hline PFHxS & 399 & 80.99 & 39 & 141 & ${ }^{18} \mathrm{O}_{2}$-PFHxS \\
\hline PFOS & 499 & 80.99 & 48 & 169 & ${ }^{13} \mathrm{C}_{2}$-PFOS \\
\hline PFOSA & 498 & 78 & 42 & 160 & ${ }^{13} \mathrm{C}_{2}$-PFOS \\
\hline 6:2Cl-PFAES & 531 & 83 & 26 & 112 & ${ }^{13} \mathrm{C}_{2}$-PFOS \\
\hline 8:2Cl-PFAES & 631 & 451 & 28 & 164 & ${ }^{13} \mathrm{C}_{2}$-PFOS \\
\hline${ }^{13} \mathrm{C}_{4}$-PFBA & 217 & 172 & 8 & 30 & \\
\hline${ }^{13} \mathrm{C}_{4}$-PFPeA & 266 & 222 & 5 & 30 & \\
\hline${ }^{13} \mathrm{C}_{2}$-PFHxA & 315 & 270 & 10 & 30 & \\
\hline${ }^{13} \mathrm{C}_{2}$-PFHpA & 367 & 322 & 24 & 47 & \\
\hline${ }^{13} \mathrm{C}_{4}$-PFOA & 417 & 372 & 7 & 39 & \\
\hline${ }^{13} \mathrm{C}_{5}$-PFNA & 468 & 219 & 16 & 45 & \\
\hline${ }^{13} C_{2}$-PFDA & 515 & 470 & 17 & 40 & \\
\hline${ }^{13} \mathrm{C}_{2}$-PFUnA & 565 & 520 & 10 & 53 & \\
\hline${ }^{13} C_{2}$-PFDoA & 615 & 570 & 10 & 53 & \\
\hline${ }^{13} C_{2}$-PFBS & 302 & $\mathbf{8 0 , 9 9}$ & 31 & 113 & \\
\hline${ }^{18} \mathrm{O}_{2}$-PFHxS & 403 & 103 & 36 & 111 & \\
\hline${ }^{13} \mathrm{C}_{2}$-PFOS & 503 & $\mathbf{8 0 , 9 9}$ & 49 & 188 & \\
\hline${ }^{13} \mathrm{C}_{8}$-PFOS & 503 & $\mathbf{8 0 , 9 9}$ & 49 & 188 & \\
\hline
\end{tabular}

$10 \%$ to $15 \%$. It might cause by the strong elution ability of high percentage acetonitrile, which decreased the resolution of analytical column. Thus, the percentage of acetonitrile at $10 \%$ was set as the optimized condition. A relatively satisfactory separation was obtained when the composition of acetonitrile increased from $10 \%$ to $45 \%$ in $1 \mathrm{~min}$, then increased to $77 \%$ in $4 \mathrm{~min}$ and finally increased to $98 \%$ in $3 \mathrm{~min}$ and was maintained for $3 \mathrm{~min}$ to allow the separations of all perfluoroalkyl acids and the elution of the longer chain homologues (such as PFHxDA and PFODA). Then, the mobile phase was rapidly returned to $10 \% \mathrm{~A}$ in $0.2 \mathrm{~min}$, followed by a conditioning step of $3.8 \mathrm{~min}$ at the starting composition before the next analysis. The final gradient elution and optimized parameters were shown in Table 1.

\subsubsection{Selection of turboflow SPE column and sample volume}

Turboflow columns provide an efficient separation of small molecules from large matrix components by creating turbulence inside the column, and macro molecules, such as proteins, can be excluded from the turboflow column [32,33]. Two types of commercial Turboflow SPE columns were compared, including silica-based Turboflow C18-P and polymeric-based Turboflow Cyclone-P columns. Both of them can retain polar and nonpolar compounds. The online analysis of a $10 \mathrm{ng} \mathrm{mL}^{-1}$ standard solution was performed on duplicate batches $(n=5)$. No peaks for PFBA or PFPeA were found using a C18-P column, and the intensities of the long chain PFAS, PFHxDA and PFODA, which were often ignored in previous literature, were strong using the Cyclone-P column, as shown in Fig. 1(c). Therefore, the Cyclone-P column was selected for further study. A large sample volume made the identification of analytes at a low concentration possible; therefore, in the present 
(a)

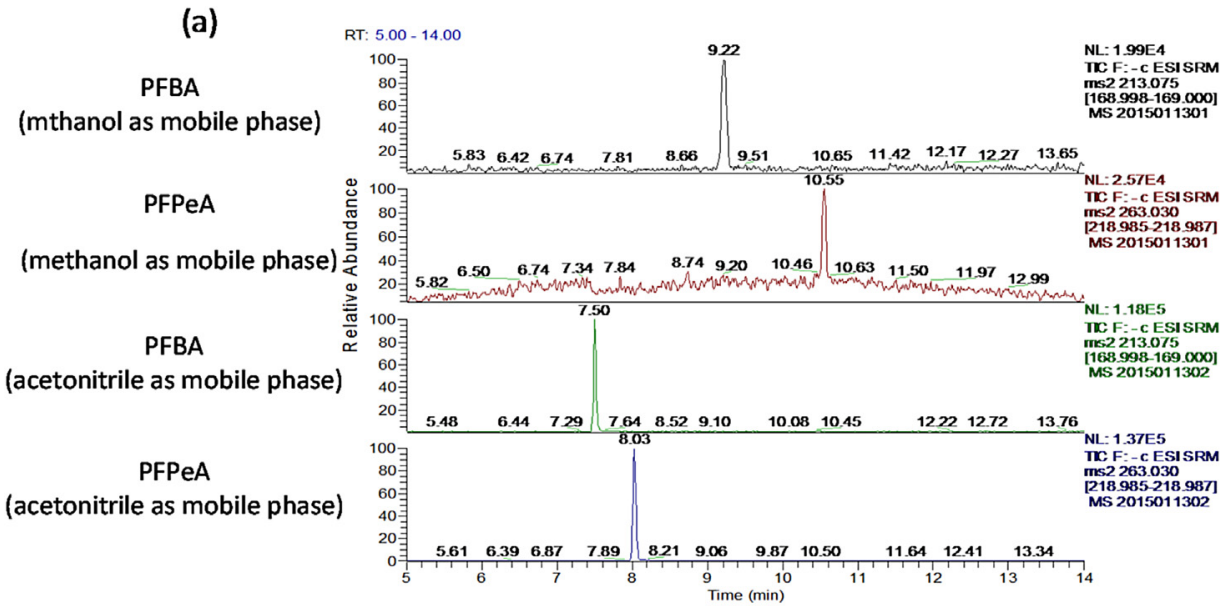

(b)

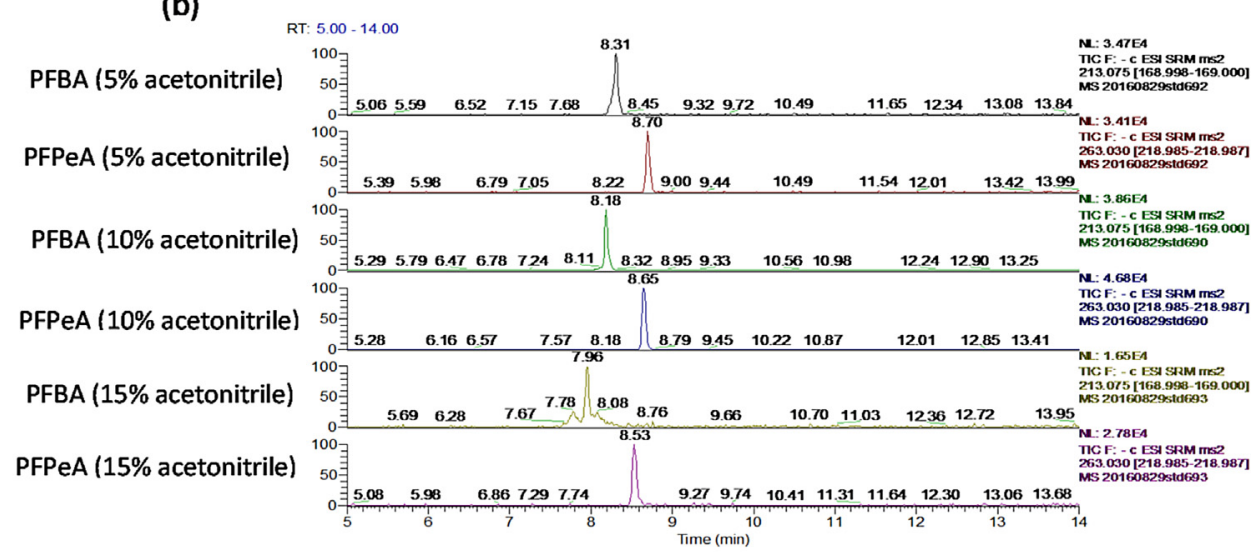

(c)

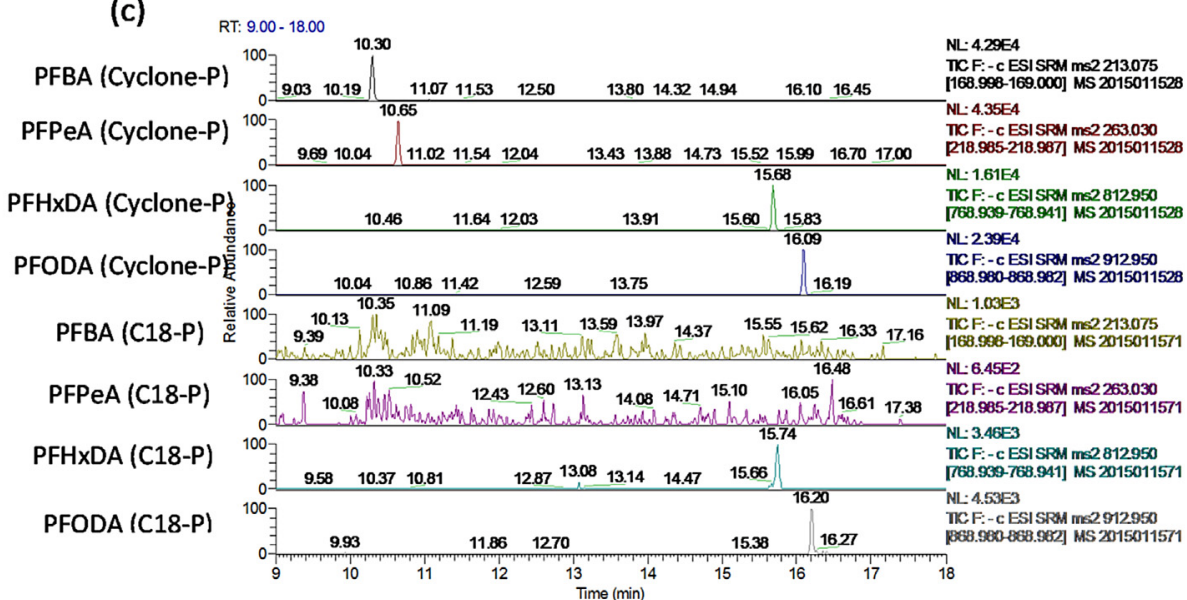

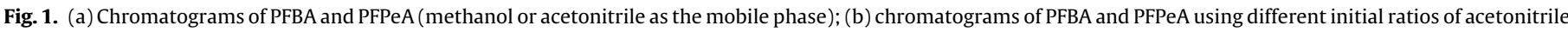

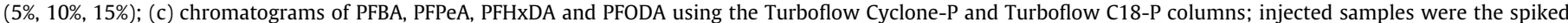
$5 \mathrm{ng} \mathrm{mL} \mathrm{m}^{-1}$ standard solutions.

study, the injected sample volume was increased from 10 to $50 \mu \mathrm{L}$. Increasing the injected sample volume resulted in sharper, higher and more symmetrical peaks for the PFASs. However, when the volume was increased to $30 \mu \mathrm{L}$, the pump pressure was obviously higher than the initial value after analysis. A sample volume of $25 \mu \mathrm{L}$ was finally selected in the present study.

\subsubsection{Loading flow rate, sample cleanup and elution time}

The flow rate during sample loading, which could affect the efficiency of the extraction and matrix elimination, was a critical parameter for the on-line Turboflow SPE procedure. Four different loading flow rates $\left(1.0,2.0,3.0\right.$ and $\left.4 \mathrm{~mL} \mathrm{~min}^{-1}\right)$ were employed to investigate the extraction efficiency (Fig. S2 in Supplementary materials). The peak intensities were highest while loading flow 


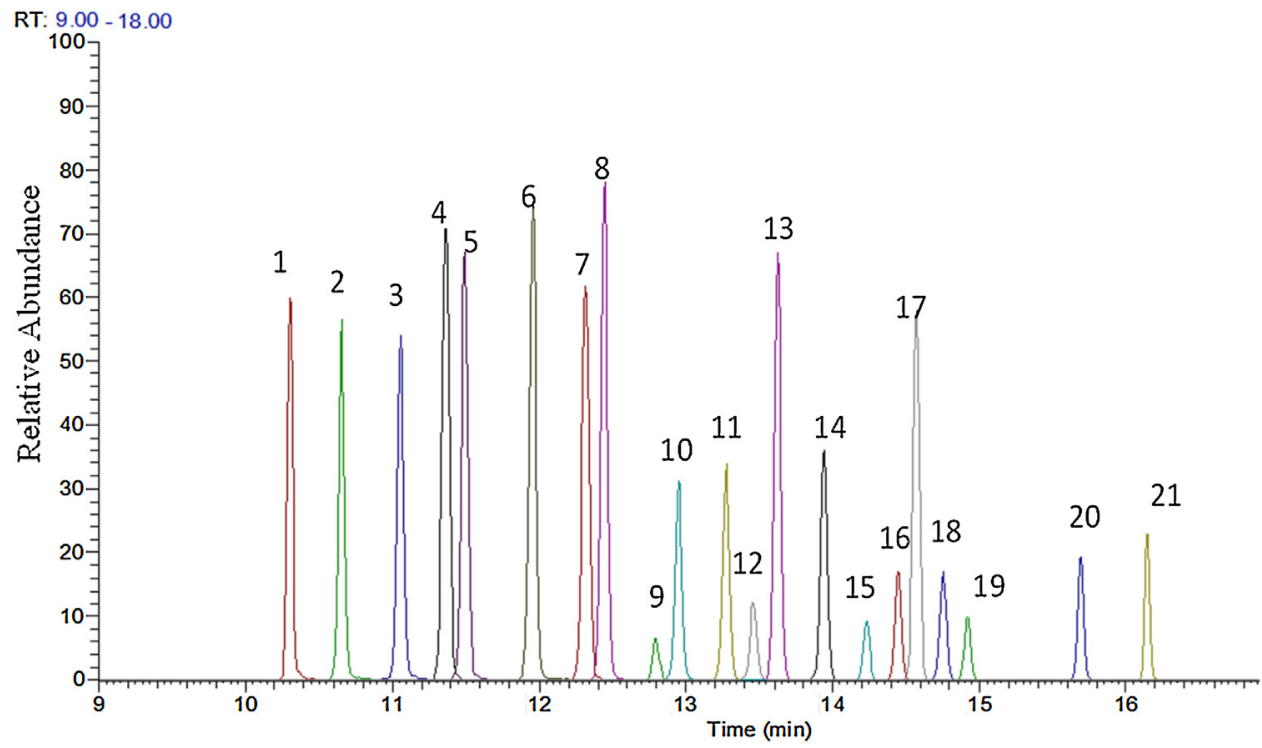

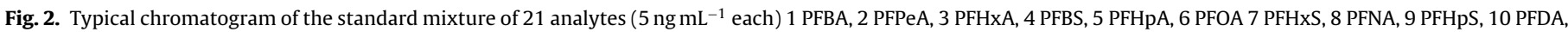
11 PFOS 12 PFUnDA, 13 F-53B, 14 PFDoA, 15 PFDS, 16 PFTrDA, 17 8:2 Cl-PFESA, 18 PFOSA, 19 PFTeDA, 20 PFHxDA, 21 PFODA.

Table 3

The linearity, LODs, spike recoveries and RSDs for the 21 PFASs in the serum samples (ng $\left.\mathrm{mL}^{-1}\right)$.

\begin{tabular}{|c|c|c|c|c|c|c|c|c|c|}
\hline \multirow[t]{2}{*}{ Analytes } & \multirow[t]{2}{*}{$r^{2}$} & \multirow[t]{2}{*}{ Linear range } & \multirow[t]{2}{*}{ LOD } & \multirow[t]{2}{*}{ LOQ } & \multicolumn{3}{|c|}{ Spike recovery $(\%) \pm \mathrm{SD}$} & \multicolumn{2}{|c|}{$\operatorname{RSD}(\%, n=5)$} \\
\hline & & & & & 0.5 & 5 & 50 & Intra-day & Inter-day \\
\hline \multicolumn{10}{|l|}{ PFCAs } \\
\hline PFBA & 0.9976 & $0.1-100$ & 0.008 & 0.025 & $114 \pm 4.3$ & $101 \pm 4.1$ & $96.9 \pm 3.5$ & 4.8 & 2.6 \\
\hline PFPeA & 0.9969 & $0.1-100$ & 0.022 & 0.066 & $103 \pm 4.6$ & $105 \pm 5.3$ & $101 \pm 7.3$ & 2.8 & 1.4 \\
\hline PFHxA & 0.9943 & $0.1-100$ & 0.032 & 0.097 & $110 \pm 5.6$ & $96.6 \pm 3.7$ & $88.3 \pm 5.2$ & 3.8 & 2.4 \\
\hline PFHpA & 0.9934 & $0.1-100$ & 0.031 & 0.094 & $113 \pm 2.7$ & $96.0 \pm 2.6$ & $86.3 \pm 5.5$ & 7.2 & 6.0 \\
\hline PFOA & 0.9906 & $0.1-100$ & 0.023 & 0.068 & $105 \pm 5.7$ & $97.4 \pm 5.4$ & $92.5 \pm 6.0$ & 2.6 & 3.1 \\
\hline PFNA & 0.9961 & $0.1-100$ & 0.036 & 0.06 & $108 \pm 5.2$ & $90.6 \pm 6.1$ & $93.5 \pm 3.6$ & 4.4 & 4.4 \\
\hline PFDA & 0.9919 & $0.1-100$ & 0.025 & 0.076 & $97.6 \pm 6.7$ & $103 \pm 2.7$ & $104 \pm 3.4$ & 6.5 & 1.1 \\
\hline PFUdA & 0.9985 & $0.1-100$ & 0.033 & 0.070 & $95.4 \pm 7.2$ & $93.5 \pm 5.5$ & $96.7 \pm 5.9$ & 2.2 & 4.6 \\
\hline PFDoA & 0.9935 & $0.1-100$ & 0.031 & 0.092 & $98.7 \pm 7.5$ & $92.1 \pm 8.3$ & $98.6 \pm 7.8$ & 8.9 & 4.8 \\
\hline PFTrA & 0.9977 & $0.1-100$ & 0.045 & 0.046 & $86.7 \pm 9.6$ & $94.7 \pm 6.9$ & $93.4 \pm 8.2$ & 5.5 & 2.4 \\
\hline PFTeA & 0.9916 & $0.1-100$ & 0.058 & 0.083 & $87.4 \pm 5.4$ & $93.5 \pm 9.2$ & $106 \pm 2.8$ & 1.5 & 4.2 \\
\hline PFHxDA & 0.9945 & $0.1-100$ & 0.15 & 0.45 & $89.7 \pm 3.6$ & $87.4 \pm 7.3$ & $87.1 \pm 5.7$ & 4.4 & 2.4 \\
\hline PFODA & 0.9909 & $0.1-100$ & 0.19 & 0.57 & $84.6 \pm 0.9$ & $85.8 \pm 2.6$ & $91.6 \pm 3.5$ & 2.6 & 1.6 \\
\hline \multicolumn{10}{|l|}{ PFSAs } \\
\hline PFBS & 0.9986 & $0.1-100$ & 0.011 & 0.032 & $106 \pm 7.2$ & $94.9 \pm 2.7$ & $90.5 \pm 0.9$ & 3.4 & 7.0 \\
\hline PFHxS & 0.9924 & $0.1-100$ & 0.053 & 0.16 & $101 \pm 8.2$ & $103 \pm 7.1$ & $94.7 \pm 7.6$ & 4.8 & 1.7 \\
\hline PFHpS & 0.9934 & $0.1-100$ & 0.062 & 0.186 & $95,2 \pm 1.1$ & $93.4 \pm 6.7$ & $98.1 \pm 3.5$ & 9.2 & 3.1 \\
\hline PFOS & 0.9922 & $0.1-100$ & 0.056 & 0.168 & $93.1 \pm 6.8$ & $102 \pm 2.7$ & $94.7 \pm 5.4$ & 2.7 & 1.3 \\
\hline PFDS & 0.9975 & $0.1-100$ & 0.053 & 0.159 & $89.4 \pm 8.8$ & $87.3 \pm 9.5$ & $93.5 \pm 4.0$ & 3.0 & 2.4 \\
\hline \multicolumn{10}{|l|}{ PFOSA } \\
\hline PFOSA & 0.9941 & $0.1-100$ & 0.034 & 0.102 & $93.1 \pm 7.1$ & $94.2 \pm 3.3$ & $91.5 \pm 4.6$ & 4.3 & 6.1 \\
\hline \multicolumn{10}{|l|}{ Cl-PFAESs } \\
\hline F-53B & 0.9967 & $0.1-100$ & 0.037 & 0.11 & $89.3 \pm 9.2$ & $93.5 \pm 5.9$ & $96.2 \pm 6.8$ & 6.3 & 2.8 \\
\hline 8:2 Cl-PFESA & 0.9903 & $0.1-100$ & 0.061 & 0.093 & $87.1 \pm 5.4$ & $90.4 \pm 4.1$ & $89.5 \pm 4.3$ & 3.0 & 4.0 \\
\hline
\end{tabular}

rate was set at $2 \mathrm{~mL} \mathrm{~min}^{-1}$, and decreased with the increasing to a higher flow rate, thus a flow rate of $2 \mathrm{~mL} \mathrm{~min}^{-1}$ was chosen.

An appropriate sample cleanup procedure can ensure optimal analytes responses as well as the elimination of the matrix components. $0.1 \%$ formic acid (mobile phase $A$ ) and acetonitrile (mobile phase B) were used to precipitate proteins [27]. In present study, $0.1 \%$ formic acid (mobile phase A) and acetonitrile(mobile phase B) with different proportions as loading solution were tested, and the peak intensity and shape of short-chain PFASs was not satisfactory, even there was no apparent peak for PFBA. Eventually, $0.1 \%$ formic acid as the only loading solution was selected as the optimized condition. And according to our subsequent monitoring data, the column pressure had no obviously change after 50 serum samples analysis, which indicated that protein was precipitated and removed efficiently. Sample cleanup times of $0.5,1.0$, and 2 min were compared (Fig. S3 in Supplementary materials). The peak response of the detected PFASs increased apparently when the cleanup time was $1.0 \mathrm{~min}$; however, the peak response of the detected PFASs decreased sharply when the time was $2 \mathrm{~min}$. Therefore, the cleanup time of 1 min was used.

After cleanup, the valve was switched to elute the analytes from the SPE column to the analytical column for a specified time. The effect of the elution time was determined for a range from 1 to $6 \mathrm{~min}$ (Fig. S4 in Supplementary materials). As the elution time increased to $5 \mathrm{~min}$, the peak intensity of the detected PFASs increased significantly, and there was no obvious improvement when the elution 
Table 4

The linearity, LODs, spike recoveries and RSDs for the 21 PFASs in the serum samples (ng $\mathrm{mL}^{-1}$ ).

\begin{tabular}{|c|c|c|c|c|c|c|c|c|c|c|c|c|c|c|c|c|}
\hline \multirow[t]{2}{*}{ Analytes } & \multicolumn{2}{|c|}{ present study } & \multicolumn{2}{|c|}{$\begin{array}{l}\text { our previous } \\
\text { off-line method }\end{array}$} & \multicolumn{2}{|c|}{$\begin{array}{l}\text { Glynn } 2012 \\
\text { (off-line) [11] }\end{array}$} & \multicolumn{2}{|c|}{$\begin{array}{l}\text { Llorca, } 2012 \\
\text { (off-line) [27] }\end{array}$} & \multicolumn{2}{|c|}{$\begin{array}{l}\text { Hanssen } 2013 \\
\text { (off-line) [23] }\end{array}$} & \multicolumn{2}{|c|}{$\begin{array}{l}\text { Kuklenyik } 2005 \\
\text { (on-line) [25] }\end{array}$} & \multicolumn{2}{|c|}{$\begin{array}{l}\text { Gosetti } 2010 . \\
\text { (on-line) [35] }\end{array}$} & \multicolumn{2}{|c|}{$\begin{array}{l}\text { Huber } 2015 \\
\text { (on-line) [1] }\end{array}$} \\
\hline & LOD & Recovery & LOD & Recovery & LOD & Recovery & LOD & Recovery & LOD & Recovery & LOD & Recovery & LOD & Recovery & LOD & Recovery \\
\hline \multicolumn{17}{|l|}{ PFCAs } \\
\hline PFBA & 0.008 & 101 & 0.035 & 87.6 & & & 0.35 & 55 & & & & & & & 0.128 & 108 \\
\hline PFPeA & 0.022 & 105 & 0.031 & 96.0 & & & 0.76 & 94 & & & 0.8 & 114 & 0.065 & 94.6 & 0.15 & 115 \\
\hline PFHxA & 0.032 & 96.6 & 0.036 & 88.4 & 0.05 & 80 & 0.14 & 120 & & & 0.6 & 114 & & & 0.055 & 108 \\
\hline PFHpA & 0.031 & 96.0 & 0.039 & 91.8 & 0.05 & 78 & 0.11 & 126 & & & 0.4 & 127 & 0.013 & 96 & 0.108 & 108 \\
\hline PFOA & 0.023 & 97.4 & 0.031 & 97.1 & 0.035 & 84 & 0.11 & 83 & 0.05 & 93 & 0.2 & 105 & 0.01 & 96.6 & 0.339 & 112 \\
\hline PFNA & 0.036 & 90.6 & 0.042 & 98.4 & 0.03 & 90 & 0.2 & 83 & 0.08 & 76 & 0.2 & 109 & & & 0.023 & 110 \\
\hline PFDA & 0.025 & 103 & 0.055 & 92.7 & 0.07 & 93 & 0.087 & 116 & & & 0.2 & 96 & 0.012 & 92.3 & 0.028 & 108 \\
\hline PFUdA & 0.033 & 93.5 & 0.057 & 87.3 & 0.05 & 92 & 0.5 & 63 & 0.05 & 62 & 0.2 & 95 & 0.038 & 92.9 & 0.032 & 108 \\
\hline PFDoA & 0.031 & 92.1 & 0.064 & 80.0 & 0.1 & 90 & 0.22 & 60 & & & 0.2 & 75 & & & 0.031 & 119 \\
\hline PFTrA & 0.045 & 94.7 & 0.19 & 53.2 & 0.15 & 81 & 0.32 & 56 & & & & & & & 0.034 & 120 \\
\hline PFTeA & 0.058 & 93.5 & 0.23 & 66.1 & 0.25 & 72 & 0.22 & 50 & & & & & & & 0.064 & 111 \\
\hline PFHxDA & 0.15 & 87.1 & 0.27 & 50.7 & & & 0.53 & 57 & & & & & & & & \\
\hline PFODA & 0.19 & 85.8 & 0.32 & 51.0 & & & 0.42 & 54 & & & & & & & & \\
\hline \multicolumn{17}{|l|}{ PFSAs } \\
\hline PFBS & 0.011 & 94.9 & 0.029 & 88.5 & 0.013 & 77 & 0.064 & 104 & & & 0.1 & 78 & 0.029 & 90.5 & 0.004 & 74 \\
\hline PFHxS & 0.053 & 95.6 & 0.045 & 96.6 & 0.007 & 81 & 0.031 & 97 & 0.03 & 66 & 0.1 & 72 & 0.026 & 96 & 0.015 & 101 \\
\hline PFHpS & 0.062 & 93.4 & 0.053 & 78.3 & & & & & & & & & & & 0.033 & 102 \\
\hline PFOS & 0.056 & 102 & 0.044 & 87.0 & 0.008 & 83 & 0.018 & 100 & 0.08 & 87 & 0.2 & 90 & 0.075 & 93.5 & 0.035 & 103 \\
\hline PFDS & 0.053 & 87.3 & 0.056 & 77.6 & 0.01 & 76 & 0.094 & 119 & & & & & & & 0.027 & 104 \\
\hline \multicolumn{17}{|l|}{ PFOSA } \\
\hline PFOSA & 0.034 & 94.2 & 0.061 & 68.9 & 0.04 & 77 & 0.026 & 112 & 0.1 & 66 & 0.05 & 95 & 0.075 & 91.9 & 0.006 & 110 \\
\hline \multicolumn{17}{|l|}{ Cl-PFAES } \\
\hline F-53B & 0.037 & 93.5 & 0.057 & 83.6 & & & & & & & & & & & & \\
\hline $8: 2 \mathrm{Cl}$-PFAES & 0.061 & 90.4 & 0.066 & 77.8 & & & & & & & & & & & & \\
\hline
\end{tabular}

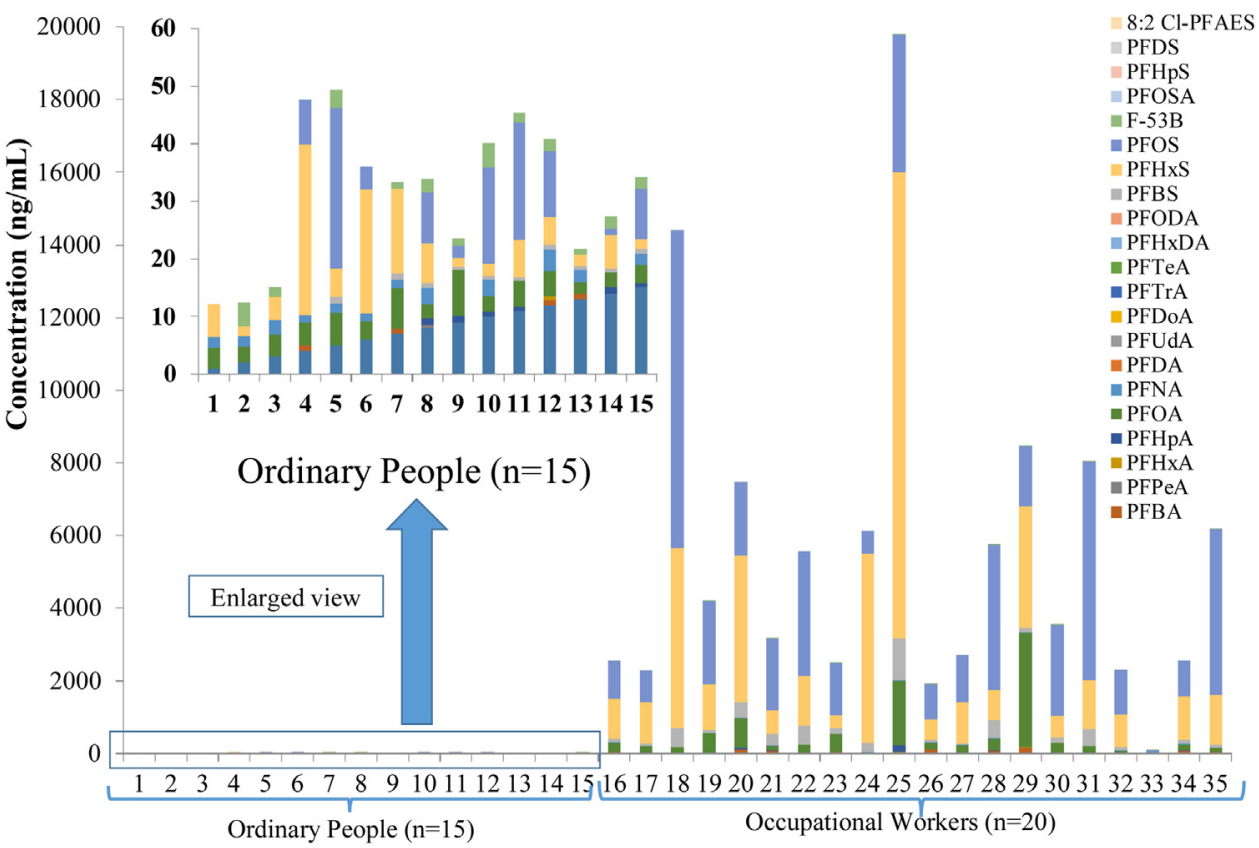

Fig. 3. Concentrations and profiles of PFASs in human serum samples (ng mL ${ }^{-1}$ ).

time was longer than $5 \mathrm{~min}$. Therefore, an elution time of 5 min was used. Fig. 2 showed a typical chromatogram of the standard mixture of 21 analytes ( $5 \mathrm{ng} \mathrm{mL}^{-1}$, each) under the optimized conditions.

\subsection{Validation of the method and performance with serum samples}

One prominent advantage of this method was the minimization of manual sample handling, which could eliminate several procedural errors and significantly decrease the analysis time. However, in contrast with the analysis of the standard solutions in acetonitrile or water, the serum samples always showed matrix effects. In present study, 13 isotope-labeled internal standards have been used to correct matrix effects on ionization efficiency as well as to compensate for variation in injection, signal variation due to ion suppression or signal enhancement $[4,5,14,16,23,26]$.

Performance standard ( $\mathrm{M}_{8} \mathrm{PFOS}$ ) was used to verify the daily dynamic range of the instrument response, namely the ionization efficiency on ESI-ion source. Blank water $(\mathrm{N}=3)$, real serum samples $(\mathrm{N}=3)$ were spiked with standard solutions prior to LC-MS/MS 
analysis. Absolute recoveries of different PFASs were shown in Table S2 in Supplementary materials. The absolute recoveries of 21 detected PFASs ranged from 87.3 to $103 \%$, with an average of 95.4\%, which indicated the low magnitude for ionization suppression. Matrix effects of most target PFASs analytes were also within the accepted range except for PFHxS, PFHpS and PFDS $(<85 \%)$ in the spiked human serum samples. However, the internal standard method were adopted for quantitative analysis in present study, the signals of isotope internal standards were suppressed/enchanced as well, thus the matrix effects were well corrected during the analysis process.

The linearity, LODs, spike recoveries and RSDs for the 21 PFASs in the serum samples (shown in Table 3), were calculated on the basis of the dilution serum. The present method displayed good linearity $\left(r^{2}>0.99\right)$ over the concentration range of $0.1-100 \mathrm{ng} \mathrm{mL}^{-1}$ for the 21 detected PFASs.

The LODs of 13 PFCAs, 5 PFSAs, PFOSA, and 2 Cl-PFESAs were 0.008 to 0.190 .011 to $0.062,0.054$, and 0.037 to $0.061 \mathrm{ng} \mathrm{mL}^{-1}$, respectively, which are considerably lower than the off-line or other on-line SPE LC-MS methods for PFASs in human serum reported in the literature (Table 4), e.g., Llorca et al. [27] (0.018-0.76 $\mathrm{ng} \mathrm{mL}^{-1}$, off-line SPE) and Gosetti et al. [35] (0.012-0.075 ng mL ${ }^{-1}$, on-line SPE). Short-chain (PFBA and PFPeA) and long-chain PFASs (PFTrA, PFTeA, PFHxDA and PFODA) were not all detected in several previous studies due to poor peak shape or blank contamination, which resulted in unsatisfactory LODs or recoveries. In the present study, the LODs of short-chain PFASs (PFBA and PFPeA) and long-chain PFASs (PFTrA, PFTeA, PFHxDA and PFODA) were 0.008, 0.022, 0.045, 0.058, 0.15 and $0.19 \mathrm{ng} \mathrm{mL}^{-1}$, respectively, which were much lower than those of previous studies [1,11,23,25,27,35], the LODs of PFBA, PFPeA, PFTrA, PFTeA, PFHxDA and PFODA were 0.35, 0.76, 0.32, 0.22, 0.53 and $0.42 \mathrm{ng} \mathrm{mL}^{-1}$, respectively, in Llorca et al.'s study [27], and the LODs of PFBA, PFPeA, PFTrA, PFTeA, PFHxDA and PFODA in our traditional MTBE method were 0.035, 0.031, 0.19, 0.23, 0.27 and $0.32 \mathrm{ng} \mathrm{mL}^{-1}$, respectively. The low LODs of short- and long-chain PFASs in the present study might result from our efforts to improve the peak shape of analytes and blank contamination control during the detection process.

The matrix spiked recoveries were obtained by spiking the serum samples of ordinary people with three levels of PFASs ( $0.5 \mathrm{ng} \mathrm{mL}^{-1}, 5 \mathrm{ng} \mathrm{mL}^{-1}$ and $50 \mathrm{ng} \mathrm{mL}^{-1}$ ), and each level of recovery experiments was repeated for three times. We have incubated the spiked standards with serum samples for one hour, keeping consistent with our previous off-line MTBE method. The spiked recoveries (spiked $5 \mathrm{ng} \mathrm{mL}^{-1}$ ) of 13 PFCAs, 5 PFSAs, PFOSA, and 2 Cl-PFESAs were $83.8-115 \%, 85.3-104 \%, 104 \%$, and $87.6-90.7 \%$, respectively, which were better than those of previous studies $[1,11,23,25,27,35]$. In particular, the recoveries of short-chain PFASs (PFBA and PFPeA) and long-chain PFASs (PFTrA, PFTeA, PFHxDA and PFODA) were 92.0, 88.5, 114, 104, 85.8, and 84.4\%, respectively, which were much better than those of previous studies $[1,11,23,25,27,35]$, recoveries of PFBA, PFPeA, PFTrA, PFTeA, PFHxDA and PFODA were 55, 94, 56, 50, 57 and 54\%, respectively, in Llorca et al.'s study [27], and recoveries of PFBA, PFPeA, PFTrA, PFTeA, PFHxDA and PFODA in our previous off-line MTBE method were $87.6,96.0,66.1,50.7$ and $51.0 \%$, respectively.

The method precision was evaluated by measuring the intraday and inter-day RSDs of replicate analyses $(n=5)$ of the $5 \mathrm{ng} \mathrm{mL}^{-1}$ standard solution under optimum conditions. The RSDs of intra-day injections (repeatability) and inter-day injections (reproducibility) ranged from $1.5 \%$ to $9.2 \%$ and from $1.1 \%$ to $7.0 \%$, respectively. All of the RSDs were below $10 \%$, which are lower than those of previous studies, including from off-line (RSDs, 5-23\%) [11,23,27] and on-line methods (RSDs, $2-17 \%$ ) $[1,25,35]$. The satisfactory precision was obtained due to the minimal sample manipulation, fewer

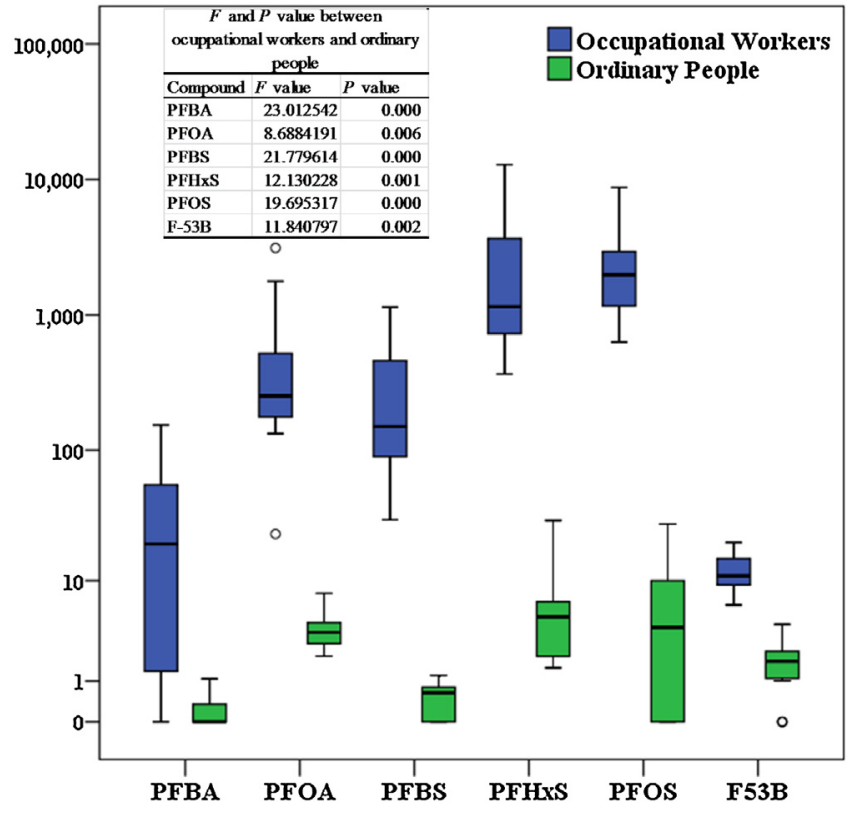

Fig. 4. Box plots of the concentrations for the main PFASs that were detected in serum samples.

sources of error, and lack of sample pretreatment. Additionally, the large particle size of the solid phase effectively eliminated the matrix components.

\subsection{Analysis of serum samples}

The present method was then applied to a population survey. Human serum samples were collected from workers of a fluorochemical manufacturing plant $(n=20)$ and ordinary adults $(n=15)$ who lived around this plant. The analytical results are listed in Table S3, S4, S5 in Supplementary materials.

The concentrations and profiles of the PFASs that were detected in the human serum samples are shown in Fig. 3. Because PFPeA, PFHxA, PFDA, PFDoDA, PFUdA, PFDoA, PFTrA, PFTeA, PFHxDA, PFODA, PFOSA, PFHpS and PFDS had low detection frequencies in all serum samples, they were not included in the following data analysis and discussion. PFBA, PFOA, PFBS, PFHxS, PFOS and F-53B were the principal PFASs in all of the serum samples, with detection frequencies above $70 \%$ and average concentrations of 19.2, 266, 148, 1266, 1417 and $7.6 \mathrm{ng} \mathrm{mL}^{-1}$, respectively. Serum PFBA, PFBS, PFHxS and PFOS were statistically higher in occupational workers than those in ordinary people $(p<0.01)$ (Fig. 4). Particularly, the average concentrations of $\mathrm{F}-53 \mathrm{~B}$ in ordinary people and occupational workers were 1.8 and $11.9 \mathrm{ng} \mathrm{mL}^{-1}$, respectively, and the detection frequencies were $80 \%$ and $100 \%$, respectively. To our knowledge, there is only one previous report of F-53B in human serum [16]. In this study, the mean concentration of F-53B in workers was lower than in high fish consumers $\left(93.7 \mathrm{ng} \mathrm{mL}^{-1}\right)$ and metal plating workers $\left(51.5 \mathrm{ng} \mathrm{mL}^{-1}\right)$, however, it was higher than that of the background control group ( $\left.4.78 \mathrm{ng} \mathrm{mL}^{-1}\right)$ [16]. Spearman's rank correlation analysis was used to test the correlation between PFOS and F-53B, and a significant positive correlation between PFOS and F-53B (Spearman's $\rho=0.68 ; p<0.01$ ) was found, which showed that they may have similar sources.

Additionally, we detected the concentration of PFASs using the traditional MTBE off-line SPE method, and the results are listed in Table S6 in Supplementary materials. The concentrations of PFBA, PFBS, PFHXS, PFOS, F-53B and PFOA that were detected using online SPE and off-line SPE were in good agreement (Fig. 5), with all of the $r$ values above $0.98(p<0.01)$. The results indicated that the on- 

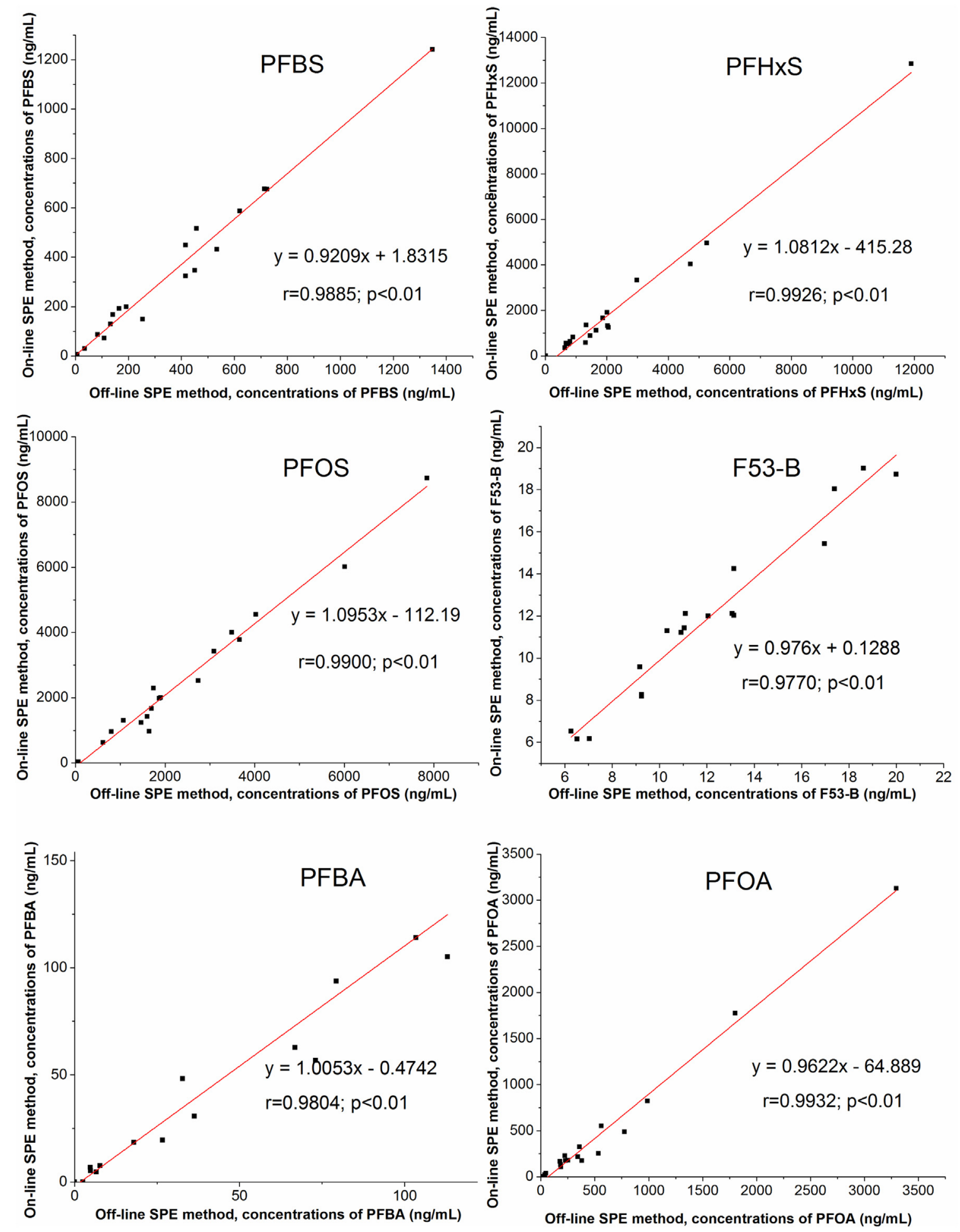

Fig. 5. Consistency comparison of the concentrations of the PFASs that were detected by on-line and off-line SPE.

line SPE method established in present study can obtain accurate data and can be applied in large scale surveys.

\section{Conclusions}

A rapid, accurate, and fully automatic method was established to simultaneously analyze 21 PFASs (with carbon chains that ranged from C4 to C18, including 13 PFCAs, 5 PFSAs, 2 Cl-PFESAs, and PFOSA) in human serum. The serum samples were directly ana- lyzed without any pre-treatment, and the LODs, accuracy, and spiked recoveries of the method satisfied the requirements for determining PFASs well. In particular, the method showed lower LODs, higher spiked recoveries for the analysis of short-chain (PFBA, PFPeA) and long-chain (PFHxDA, PFODA) PFASs than both off-line and on-line methods from previous studies. Moreover, the concentrations of the PFASs that were detected by the present on-line SPE method were consistent with the traditional off-line SPE method ( $r$ : 0.98-0.99). The procedures of the present method were greatly 
simplified and achieved a fully automatic detection of PFASs, with an analysis time of only 20 min per sample. The method has good practical prospects for the large scale investigation of trace PFASs in human serum in a rapid, accurate, and facile manner.

\section{Acknowledgements}

This study was jointly supported by the Chinese Academy of Sciences (XDB14030500), and the National Natural Science Foundation $(21477154,21321004,21277164$, and 21177146). The authors would also like to thank all of the blood donors.

\section{Appendix A. Supplementary data}

Supplementary data associated with this article can be found, in the online version, at http://dx.doi.org/10.1016/j.chroma.2016.09. 050 .

\section{References}

[1] S. Huber, J. Brox, An automated high-throughput SPE micro-elution method for perfluoroalkyl substances in human serum, Anal. Bioanal. Chem. 407 (2015) 3751-3761

[2] Y. Shi, R. Vestergren, Z. Zhou, X. Song, L. Xu, Y. Liang, Y. Cai, Tissue distribution and whole body burden of the chlorinated polyfluoroalkyl ether sulfonic acid F-53B in crucian carp (Carassius carassius): evidence for a highly bioaccumulative contaminant of emerging concern, Environ. Sci. Technol. 49 (2015) 14156-14165.

[3] C. Lau, K. Anitole, C. Hodes, D. Lai, A. Pfahles-Hutchens, J. Seed, Perfluoroalkyl acids: a review of monitoring and toxicological findings, Toxicol. Sci. 99 (2007) 366-394.

[4] Y. Gao, J. Fu, H. Cao, Y. Wang, A. Zhang, T. Wang, Y. Liang, C. Zhao, G. Jiang, Differential accumulation and elimination behavior of perfluoroalkyl acid isomers in occupational workers in a manufactory in China, Environ. Sci. Technol. 49 (2015) 6953-6962.

[5] J. Fu, Y. Gao, T. Wang, Y. Liang, A. Zhang, Y. Wang, G. Jiang, Elevated levels of perfluoroalkyl acids in family members of occupationally exposed workers: the importance of dust transfer, Sci. Rep. 5 (2015).

[6] A. Calafat, L. Wong, Z. Kuklenyik, J. Reidy, L. Needham, Polyfluoroalkyl chemicals in the US population: data from the national health and nutrition examination survey (NHANES) 2003-2004 and comparisons with NHANES 1999-2000, Environ. Health Perspect. (2007) 1596-1602.

[7] L. Tao, J. Ma, T. Kunisue, E.L. Libelo, S. Tanabe, K. Kannan, Perfluorinated compounds in human breast milk from several Asian countries, and in infant formula and dairy milk from the United States, Environ. Sci. Technol. 42 (2008) 8597-8602

[8] J. Li, F. Guo, Y. Wang, J. Zhang, Y. Zhong, Y. Zhao, Y. Wu, Can nail, hair and urine be used for biomonitoring of human exposure to perfluorooctane sulfonate and perfluorooctanoic acid? Environ. Int. 53 (2013) 47-52.

[9] Y. Zhao, H. Wan, A. Law, X. Wei, Y. Huang, J. Giesy, M. Wong, C. Wong, Risk assessment for human consumption of perfluorinated compound-contaminated freshwater and marine fish from Hong Kong and Xiamen, Chemosphere 85 (2011) 277-283.

[10] H. Peng, K. Zhang, Y. Wan, J. Hu, Tissue distribution, maternal transfer, and age-related accumulation of dechloranes in Chinese sturgeon, Environ. Sci. Technol. 46 (2012) 9907-9913.

[11] A. Glynn, U. Berger, A. Bignert, S. Ullah, M. Aune, S. Lignell, P. Darnerud, Perfluorinated alkyl acids in blood serum from primiparous women in Sweden: serial sampling during pregnancy and nursing, and temporal trends 1996-2010, Environ. Sci. Technol. 46 (2012) 9071-9079.

[12] Z. Wang, I. Cousins, M. Scheringer, R. Buck, K. Hungerbühler, Global emission inventories for C4-C14 perfluoroalkyl carboxylic acid (PFCA) homologues from 1951 to 2030, Part I: production and emissions from quantifiable sources, Environ. Int. 70 (2014) 62-75.

[13] M. Gomis, Z. Wang, M. Scheringer, I. Cousins, A modeling assessment of the physicochemical properties and environmental fate of emerging and novel per- and polyfluoroalkyl substances, Sci. Total Environ. 505 (2015) 981-991.

[14] T. Ruan, Y. Lin, T. Wang, R. Liu, G. Jiang, Identification of novel polyfluorinated ether sulfonates as PFOS alternatives in municipal sewage sludge in China, Environ. Sci. Technol. 49 (2015) 6519-6527.

[15] S. Wang, J. Huang, Y. Yang, Y. Hui, Y, Ge, T. Larssen, G. Yu, S. Deng, B. Wang, C. Harman, First report of a Chinese PFOS alternative overlooked for 30 years: its toxicity, persistence, and presence in the environment, Environ. Sci. Technol. 47 (2013) 10163-10170.
[16] Y. Shi, R. Vestergren, L. Xu, Z. Zhou, C. Li, Y. Liang, Y. Cai, Human exposure and elimination kinetics of chlorinated polyfluoroalkyl ether sulfonic acids (Cl-PFESAs), Environ. Sci. Technol. 50 (2016) 2396-2404.

[17] F. Pérez, M. Nadal, A. Navarro-Ortega, F. Fàbrega, J. Domingo, D. Barceló, M. Farré, Accumulation of perfluoroalkyl substances in human tissues, Environ. Int. 59 (2013) 354-362.

[18] J. Sun, P. Hui, J. Hu, Temporal trends of polychlorinated biphenyls, polybrominated diphenyl ethers, and perfluorinated compounds in Chinese sturgeon (Acipenser sinensis) eggs (1984-2008), Environ. Sci. Technol. 49 (2015) 1621-1630

[19] A. Glynn, U. Berger, A. Bignert, et al., Perfluorinated alkyl acids in blood serum from primiparous women in Sweden: serial sampling during pregnancy and nursing, and temporal trends 1996-2010, Environ. Sci. Technol. 46 (2012) 9071-9079.

[20] B. Gewurtz, O. De Silva, M. Backus, J. McGoldrick, J. Keir, J. Small, L. Melymuk, G. Muir, Perfluoroalkyl contaminants in Lake Ontario Lake Trout: detailed examination of current status and long-term trends, Environ. Sci. Technol. 46 (2012) 5842-5850.

[21] Z. Wang, T. Cousins, M. Scheringer, K. Hungerbühler, Fluorinated alternatives to long-chain perfluoroalkyl carboxylic acids (PFCAs), perfluoroalkane sulfonic acids (PFSAs) and their potential precursors, Environ. Int. 60 (2013) 242-248.

[22] M. Hirata-Koizumi, S. Fujii, K. Hina, M. Matsumoto, M. Takahashi, A. Ono, A. Hirose, Repeated dose and reproductive/developmental toxicity of long-chain perfluoroalkyl carboxylic acids in rats: perfluorohexadecanoic acid and perfluorotetradecanoic acid, Fundam. Toxicol. Sci. 2 (2015) 177-190.

[23] L. Hanssen, A. Dudarev, S. Huber, J. Odland, E. Nieboer, T. Sandanger, Partition of perfluoroalkyl substances (PFASs) in whole blood and plasma, assessed in maternal and umbilical cord samples from inhabitants of arctic Russia and Uzbekistan, Sci. Total Environ. 447 (2013) 430-437.

[24] L. Haug, C. Thomsen, G. Becher, A sensitive method for determination of a broad range of perfluorinated compounds in serum suitable for large-scale human biomonitoring. J. Chromatogr. A 1216 (2009) 385-393.

[25] Z. Kuklenyik, L. Needham, A. Calafat, Measurement of 18 perfluorinated organic acids and amides in human serum using on-line solid-phase extraction, Anal. Chem. 77 (2005) 6085-6091.

[26] F. Perez, M. Llorca, M. Farré, D. Barceló, Automated analysis of perfluorinated compounds in human hair and urine samples by turbulent flow chromatography coupled to tandem mass spectrometry, Anal. Bioanal. Chem. 402 (2012) 2369-2378.

[27] M. Llorca, F. Pérez, M. Farré, S. Agramunt, M. Kogevinas, D. Barceló, Analysis of perfluoroalkyl substances in cord blood by turbulent flow chromatography coupled to tandem mass spectrometry, Sci. Total Environ. 433 (2012) $151-160$.

[28] S. Huber, L. Ahrens, B. Bårdsen, U. Siebert, J. Bustnes, G. Víkingsson, R. Ebinghaus, D. Herzke, Temporal trends and spatial differences of perfluoroalkylated substances in livers of harbor porpoise (Phocoena phocoena) populations from Northern Europe, 1991-2008, Sci. Total Environ. 419 (2012) 216-224

[29] Y, Y. Shi, L. Pan, Liang, Y. Cai, An on-line solid phase extraction-liquid chromatography-tandem mass spectrometry method for the determination of perfluoroalkyl substances in the Antarctic ice core samples, Chin. Chem. Lett. 26 (2015) 1073-1078

[30] I. Ezkurdia, J. Vaízquez, A. Valencia, M. Tress, Analyzing the first drafts of the human proteome, J. Proteome Res. 13 (2014) 3854-3855.

[31] S. Rayne, K. Forest, Perfluoroalkyl sulfonic and carboxylic acids: a critical review of physicochemical properties, levels and patterns in waters and wastewaters, and treatment methods, J. Environ. Sci. Health Part A 44 (2009) 1145-1199.

[32] F. Guo, Q. Liu, J. Shi, F. Wei, G. Jiang, Direct analysis of eight chlorophenols in urine by large volume injection on-line turbulent flow solid-phase extraction liquid chromatography with multiple wavelength ultraviolet detection, Talanta 119 (2014) 396-400.

[33] F. Guo, J. Shao, Q. Liu, J. Shi, G. Jiang, Automated and sensitive determination of four anabolic androgenic steroids in urine by on-line turbulent flow solid-phase extraction coupled with liquid chromatography-tandem mass spectrometry: a novel approach for clinical monitoring and doping control, Talanta 125 (2014) 432-438.

[34] G. Shan, Z. Wang, L. Zhou, P. Du, X. Luo, Q. Wu, L. Zhu, Impacts of daily intakes on the isomeric profiles of perfluoroalkyl substances (PFASs) in human serum, Environ. Int. 89 (2016) 62-70.

[35] F. Gosetti, U. Chiuminatto, D. Zampieri, E. Mazzucco, E. Robotti, G. Calabrese, M.C. Gennaro, E. Marengo, Determination of perfluorochemicals in biological, environmental and food samples by an automated on-line solid phase extraction ultra high performance liquid chromatography tandem mass spectrometry method, J. Chromatogr. A 1217 (2010) 7864-7872. 